\title{
BMJ Open Dynamic changes of circulating tumour DNA in surgical lung cancer patients: protocol for a prospective observational study
}

\author{
Kezhong Chen, ${ }^{1}$ Heng Zhao, ${ }^{1}$ Fan Yang, ${ }^{1}$ Bengang Hui, ${ }^{1}$ Tianyang Wang, ${ }^{2}$ \\ Lieu Tu Wang, ${ }^{2}$ Yanbin Shi, ${ }^{2}$ Jun Wang ${ }^{1}$
}

To cite: Chen K, Zhao H, Yang $\mathrm{F}$, et al. Dynamic changes of circulating tumour DNA in surgical lung cancer patients: protocol for a prospective 2018;8:e019012. doi:10.1136/ bmjopen-2017-019012

- Prepublication history for this paper is available online. To view these files, please visit the journal online (http://dx.doi. org/10.1136/bmjopen-2017019012).

Received 7 August 2017 Revised 2 November 2017 Accepted 28 November 2017 observational study. BMJ Open

\section{ABSTRACT}

Introduction Circulating tumour DNA (ctDNA) has potential applications in cancer management. Most previous studies about ctDNA focused on advanced stage cancer patients. We have completed a clinical prospective study (NCT02645318) and showed the feasibility and clinical application of ctDNA detection in early stage nonsmall cell lung cancer (NSCLC) patients. The aim of this study is to investigate the elimination rate of ctDNA level after surgery. This is the first prospective study to evaluate the perioperative dynamic changes of ctDNA in surgical lung cancer patients.

Methods and analysis This is a prospective observational study to determine the elimination rate of circulating tumour DNA after surgery. Consecutive patients with suspected lung cancer who undergo curative-intent lung resection will be enrolled. $10 \mathrm{~mL}$ blood samples are taken by intravenous puncture. Plasma samples are obtained before surgery (time A) and at a series of scheduled time-points ( 2 min to 72 hours, time B to F) after tumour resection. DNA is prepared from $4 \mathrm{~mL}$ of purified plasma. A multiplex assay based on circulating singlemolecule amplification and resequencing technology (CSMART) is used to simultaneously detect and quantitate hot spot EGFR, KRAS, BRAF, ERBB2, PIK3CA, TP53, ALK, RET and MET plasma DNA variants. Positive plasma mutations are validated in tumour tissue and normal lung tissue by targeted sequencing.

Ethics and dissemination Ethical approval has been obtained from the Peking University People's Hospital Medical Ethics Committee (2016PHB156-01). Results will be disseminated through presentations at scientific meetings and publications in peer-reviewed journals. Trial registration number NCT02965391; Pre-results.

Check for updates

${ }^{1}$ Department of Thoracic Surgery, Peking University People's Hospital, Beijing, China ${ }^{2}$ Berry Genomics Corp, Beijing, China

Correspondence to

Dr Jun Wang;

wangjun@pkuph.edu.cn

\section{INTRODUCTION}

Circulating tumour DNA (ctDNA) released from multiple tumour regions and is thereby overcome tumour heterogeneity. Identifying ctDNA offers the opportunity to assist cancer screening or earlier diagnosis, to detect molecular profiling, to monitor disease progress and predict treatment response. ${ }^{1}$ However, most previous studies of ctDNA
Strengths and limitations of this study

- This is the first prospective study to evaluate the dynamic changes of ctDNA in early stage, surgical lung cancer patients.

- Plasma samples are obtained critically followed the scheduled time-point, which we believe to be both robust and accurate and so sets a convincible result for clinical application.

- We have completed a series of studies with solid data of circulating tumour DNA detection in surgical non-small cell lung cancer patients, including the concordance between plasma and tumour tissue DNA, the preliminary exploration of ctDNA for early diagnosis, and a novel technique to simultaneously detect and quantitate multiplex hot spot plasma DNA variants.

- The weaknesses of this study is that it is a single institution clinical trial so that there is a bias in the patient's selection.

have focused on advanced stage or metastatic cancers (stage IIIB-IV). ${ }^{2-4}$ Few data are available to indicate whether ctDNA analyses is applicable for stage I-IIIA non-small-cell lung cancer (NSCLC) patients, ${ }^{5}$ largely because the low level of early stage disease makes detection of ctDNA challenging. ${ }^{6}$

Furthermore, there are fewer data available on the stability of cfDNA. Lo et al investigated the clearance of circulating fetal DNA after delivery by using quantitative PCR analysis and showed the mean half-life for circulating fetal DNA was $16.3 \mathrm{~min} .{ }^{7}$ Nevertheless, in cancer patients, the mechanism of DNA clearance from plasma is poorly understood and it is not known how surgery influence ctDNA release and clearance. Diehl's group quantified ctDNA in 18 stage IV colorectal cancer patients undergoing multimodality therapy for colorectal cancer, and found a sharp drop in the ctDNA level by the day of discharge (two to ten days after surgery) in all 


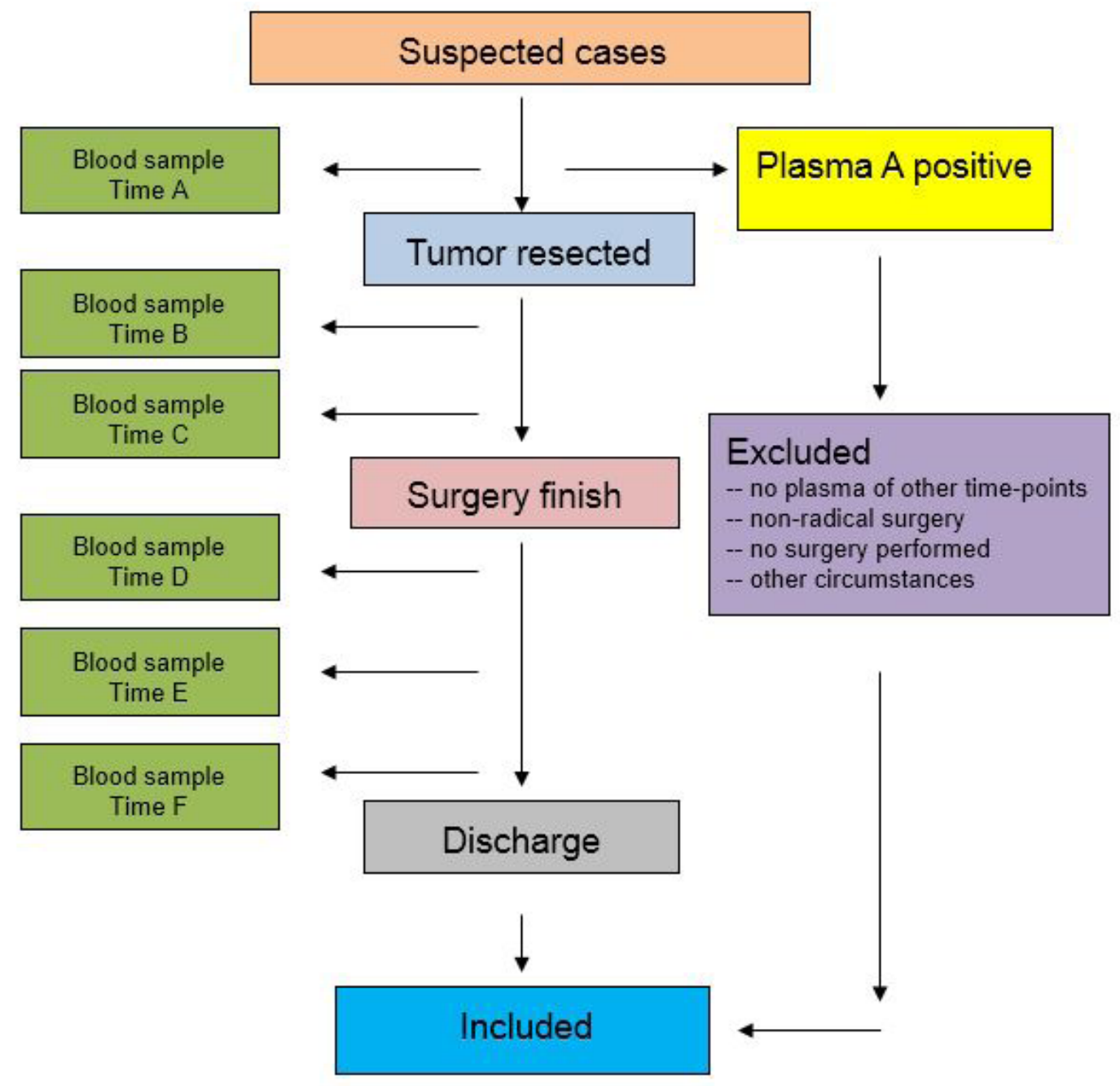

Figure 1 Flow diagram.

subjects. Through evaluation of a subject whose plasma were sampled at multiple early times after complete resection, they estimated the half-life of ctDNA after surgery as $114 \mathrm{~min} .{ }^{8}$ Due to the small sample size, the eliminate rate of ctDNA in cancer patients is still unclear. ${ }^{9}$ What's more, none of the studies have investigated the clearance in early stage lung cancer patients.

In contrast with other common solid tumours such as breast and colon cancer, survival after radical resection of early-stage NSCLC are poor, with a significant proportion $(20 \%-40 \%)$ of recurrence rate. ${ }^{10}$ Since it's dissatisfied to predict prognosis with available clinical pathological characteristics, many attempts have been made to develop biomarkers that could provide prognostic information. However, in practice this approach has been very challenging - many studies have yielded prognostic signatures, but they seldom overlap with other signatures, with methods that might not be transferable to real-life clinical situations. ${ }^{11}$

\section{Rationale for the study}

Following surgery or treatment with curative intent, data from proof-of-concept studies have confirmed detection of ctDNA may signal the presence of minimal residual disease (MRD) even in the absence of any other clinical evidence of disease, and the presence of ctDNA could identify patients who may be at higher risk of relapse. ${ }^{612}$ However, no studies explored whether the detection of ctDNA after surgery was due to the MRD or incomplete clearance. It's meaningful to know when the plasma ctDNA completely decay after R0 tumour resection, at which time the ctDNA level can be used as a baseline for postoperative surveillance.

Therefore, this is the first study to investigate the perioperative dynamic changes of ctDNA in surgical lung cancer patients. With the accurate evaluation of ctDNA clearance after tumour resection, we will explore the most appropriate time to detect ctDNA for postoperative surveillance.

\section{Previous work}

Since the feasibility for ctDNA detection for early stage NSCLC was under the concern, we performed a prospective study which enrolled 76 consecutive lung cancer patients comparing a panel of 50 cancer-related genetic mutations between plasma ctDNA and tumour tissue 


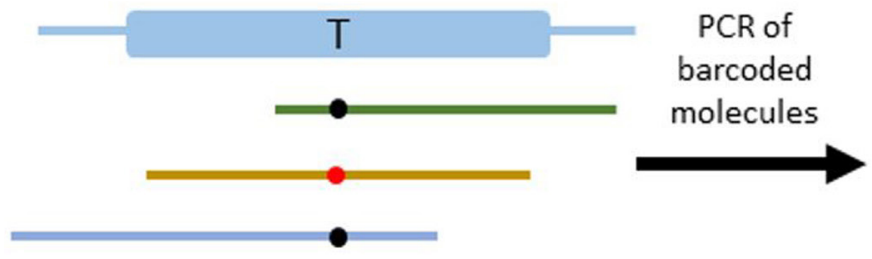

Individual DNA molecules in plasma

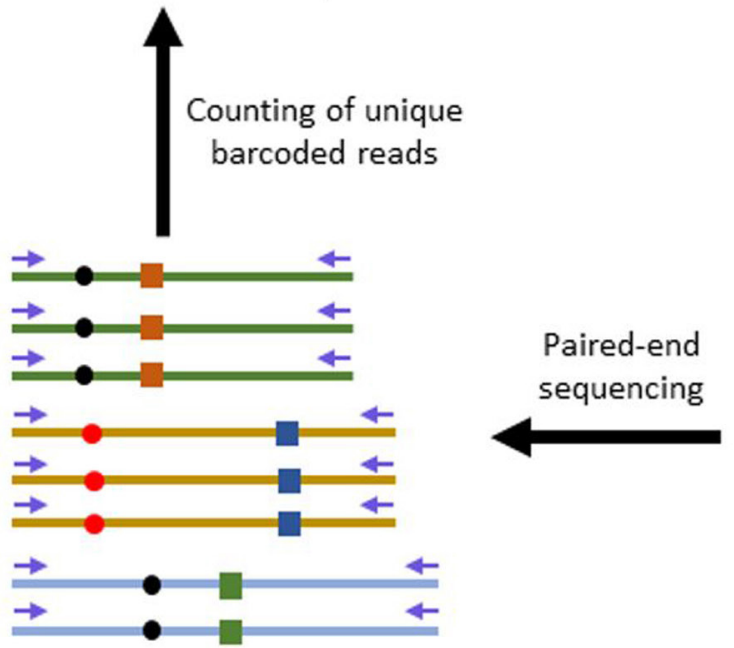

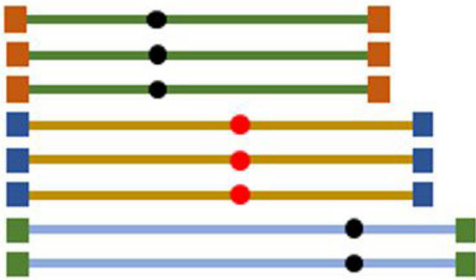

Inverse PCR of

circularised DNA

molecules

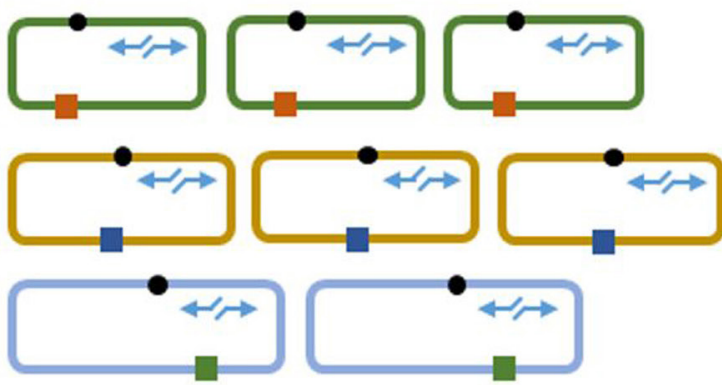

Figure 2 Concept of cSMART for quantification of allelic variants in plasma.

DNA. The result demonstrated that the concordance of ctDNA and tumour tissue DNA was nearly $70 \%$ in these surgical NSCLC patients. To explore the potential application for early diagnosis, we also compared ctDNA to tumour protein markers (carcinoembryonic antigen (CEA), carbohydrate antigen 19-9 (CA19-9), carbohydrate antigen 125 (CA125), cytokeratin 19 fragment (CYFRA21-1), neuron-specific enolase (NSE)) and radiographic-based clinical predictive models. More patients were positive as assayed by ctDNA $(48,63.2 \%)$ than with serum tumour protein markers $(36,49.3 \%)$. The area under the curve was higher in ctDNA $(0.887,95 \% \mathrm{CI}$ 0.788 to 0.986 ) than in the two clinical prediction models $(0.803,95 \%$ CI 0.647 to $0.959 ; 0.69,95 \%$ CI 0.512 to 0.869$)$ for estimating malignancy of solitary pulmonary nodules. Besides, ctDNA mutation frequency 1 day after surgery was significantly lower than before surgery $(0.28 \%$ vs $7.94 \%$, $\mathrm{P}<0.001$ ), but only some of the mutations decreased to zero. This previous study confirmed ctDNA detection in early stage NSCLC patients is feasible and presents a potential application for clinical management. ${ }^{13}$

\section{Hypotheses}

1. For patient with surgical lung cancer, ctNDA will decline rapidly after surgery.

2. The ctDNA level 72 hours after surgery can be used as a reference value for postoperative surveillance.

3. Correlation exists between clinical features and ctDNA level after surgery.
Primary aims

1. To investigate the perioperative dynamic changes of ctDNA in surgical lung cancer patients.

2. To determine the appropriate time point of ctDNA detection for postoperative surveillance.

\section{Secondary aims}

1. To compare the genetic detection between tumour tissue and plasma ctDNA in surgical lung cancer patients.

2. To explore the relationship between clinical features and perioperative ctDNA level.

3. To investigate the correlation between circulating tumour DNA level before and after surgery with tumour recurrence.

\section{METHODS AND ANALYSIS}

This protocol outlines a prospective clinical trial in which surgical lung cancer patients will be recruited and ctDNA level will be assessed in patient's plasma at multiple time points. Patients will also be followed after surgery.

\section{Study population}

The population will be drawn from Peking University People's Hospital Thoracic Surgery Department. All of the patients will receive contrast-enhanced thoracic CT scans, abdominal and adrenal gland ultrasonography or CT, brain MRI or CT and a bone scan before surgery. 
$\mathrm{PET} / \mathrm{CT}$ is not mandatory in every patient. Consecutive patients who are suspected stage I- IIIA NSCLC before surgery will be eligible and included in this study, if they have no malignant tumour history within the past 5 years, the lesions are not pure ground glass opacity, and willing to write informed consent. The tumours will be classified according to the eighth American Joint Committee on Cancer (AJCC) TNM staging system. The exclusion criteria are as followed: (1) Multiple primary lung cancer; (2) R1, R2 resection; (3) Histology is not NSCLC; (4) Unqualified blood samples.

Follow-up information will be obtained through clinic follow-up notes 1 month after surgery and every 3 months thereafter. The follow-up protocol consists of chest CT scan and abdominal ultrasound performed every 6 months for the first 2 years and yearly thereafter. Brain MRI and bone scan are performed every year for 5 years or any time the patient has symptoms.

Clinical and demographic data will be collected including age, sex, smoking status, surgical method (wedge resection first, followed by lobectomy or lobectomy directly), tumour location, histology, postoperative TNM-staging, and the exact time of each blood sample extracted. Follow-up data will also be collected.

\section{Sampling schedule}

$10 \mathrm{~mL}$ blood samples are taken by intravenous puncture. Plasma samples are obtained before surgery (time A) and at a series of scheduled time-points (time B: 5 min; time C: 30 min; time D: 2 hour; time E: 1 day; time F: 3 days) after tumour resection. According to the different surgical method, the time-points will be recorded discriminatively. If the patient receives wedge resection first, time $B$ will be recorded just $5 \mathrm{~min}$ after wedge resection. If the patient receives lobectomy directly, time $\mathrm{B}$ will be recorded $5 \mathrm{~min}$ after the pulmonary vein being cut-off. If the patient has pleural metastasis or is unable to get a R0 resection, he/ she will be excluded from this study (figure 1).

Peripheral blood samples obtained at each time point will be processed to isolate plasma within 2 hours from EDTA tubes. Samples are centrifuged for $10 \mathrm{~min}$ at $1600 \mathrm{x} g$ and supernatants from these samples are again centrifuged at $16000 \times \mathrm{g}$ for $10 \mathrm{~min}$. Plasma and peripheral blood lymphocyte debris are collected and stored at $-80^{\circ} \mathrm{C}$ until needed. ctDNA is prepared from $4 \mathrm{~mL}$ of purified plasma and will be extracted immediately to avoid DNA degradation.

We designed a multiplex assay based on circulating single-molecule amplification and resequencing technology (cSMART) to simultaneously detect and quantitate hot spot EGFR, KRAS, BRAF, ERBB2, PIK3CA, TP53, ALK, RET and MET plasma DNA variants. ${ }^{14}{ }^{15}$ cSMART is capable to detect ctDNA with minimum VAF (variant allele frequency) of $0.01 \%$, which has been verified by serial dilution of ctDNA standards detection. Positive plasma mutations (time A plasma) are validated in tumour tissue and normal lung tissue by targeted sequencing. Tumour tissue samples and normal tissue samples will be collected during surgery when a frozen section is confirmed as malignant.

Enrolled patients will be followed after surgery, whose blood sample will be obtained each 3 months at their postoperative review to detect the eight hot spot DNA variants.

\section{Isolation of DNA and RNA from specimens}

Isolation of genomic DNA and total RNA from FPPE specimen blocks are performed using the AllPrep DNA/RNA FFPE Kit (Qiagen, Hilden, Germany). By spectroscopy analysis, all purified DNA and RNA samples are judged to be of high quality for mutation analysis. Ten-millilitre blood samplesare taken by venipuncture at each timepoints. DNA for cSMART assay is prepared from $4 \mathrm{~mL}$ of purified plasma using the QIAamp Circulating Nucleic Acid Kit (Qiagen, Hilden, Germany). The concentration of the purified DNA is measured by the Qubit dsDNA HS Assay Kit (Life Technologies, Eugene, OR).

\section{Plasma and tissue mutation assays}

The cSMART assay designed specifically for detection of oncogenic plasma mutations was performed as previously described, ${ }^{15}$ and modified for simultaneous detection and quantitation of hot spot oncogenic mutations in plasma (figure 2). Cell free DNA extracted from $4 \mathrm{~mL}$ of plasma is used to construct DNA libraries. DNA molecules are end modified by 15 cycles of PCR using the high fidelity DNA polymerase (Promega, Madison, WI) with a low sequencing error rate of approximately 1 in $10^{5}$ nucleotides. A bridging oligonucleotide is specifically designed with a four-base NNNN code and synthesised as mixture of degenerate DNA molecules, which serve to randomly and uniquely barcode every single plasma molecule in the library. Hot spot mutations within the circularised single-strand molecules are then amplified by multiple inverse PCR using primer pairs strategically located 20 to $48 \mathrm{bp}$ from each mutation loci to ensure maximum sensitivity and specificity for mutation detection. The pool of allelic molecules generated by inverse PCR is subjected to paired end sequencing on the MiSeq platform (Illumina, San Diego, CA). A minimum of one unique read is used to define a read group, while duplicate or higher-order reads with the same start and stop positions are distinguished by their unique barcodes and only counted once to correct for PCR bias. The abundance of each mutation is expressed as the number of mutant molecules per $4 \mathrm{~mL}$ of plasma.

Tilling PCR amplification sequencing (tPAS) is used to detect mutations in tissue samples. In brief, gDNA purified from frozen tissue samples are PCR amplified by multiple primers targeting exon regions of EGFR, KRAS, BRAF, ERBB2, PIK3CA, TP53, RNA splicing sites and exon regions of MET; and RNA is reverse transcribed to cDNA followed by PCR amplification with primers targeting multiple fusion variants 
of ALK and RET. The PCR products are subjected to library construction which are then paired-end sequenced by MiSeq (Illumina, San Diego, CA). The publicly available FASTQ-join programme (https:// expressionanalysis.github.io/ea-utils) is then used to reconstruct the overlapping paired end sequences to generate contiguous consensus sequences for each allelic molecule. The sequencing reads are aligned to the reference genome using BWA for mutation calling.

The known mutations specifically targeted in the study are G719A/C/S (three variants, G719X), exon 19 deletions (16 variants), exon 20 insertions (three variants), S768I, T790M, C797S, exon 21 point mutations L858R and L861Q in EGFR; G12A/C/D/R/S/V (six variants, G12X), G13D, Q61E/H/K/L/P/R (six variants, Q61X), and $\mathrm{A} 146 \mathrm{~T} / \mathrm{P} / \mathrm{V}$ (three variants, A146X) in KRAS; exon 20 insertions (five variants) in ERBB2 and V600D/E/K/R (four variants, V600X) in BRAF; R88Q, E542K, E545K/D (two variants, E545X) and A1047R/L (two variants, A1047X) in PIK3CA; R175C/H (two variants, R175X), R248W/Q (two variants, R248X) and R273C/H (two variants, R273X) in TP53; MET exon 14 skipping and ALK, RET fusions. On the basis of the positioning of targeting primers within the exonic sequences of the EGFR, KRAS, ERBB2, BRAF, PIK3CA, TP53, MET, RET and ALK genes, the cSMART assay is also designed for detection of novel mutations.

\section{Sample size calculation}

According to the previous study, the frequency of these eight driver mutations(EGFR, KRAS, BRAF, ALK, HER2, PIK3CA, RET, MET) are about $40 \%$ in Chinese lung cancer patients. ${ }^{1617}$ Our study ${ }^{18}$ has showed the sensitivity of tissue mutation detected in ctDNA is about $50 \%$. Therefore, the included proportion of all screening patients would be 1:5. Previous studies for clearance of ctDNA (include fetal DNA and Epstein-Barr virus DNA) were from 10 to 20 subjects, ${ }^{7819}$ therefore, we intend to enrol 20 patients for analysis. Considered the complicated clinical events, $20 \%$ patients may have to be excluded due to the difficulty of obtaining samples at expected time point. So the overall recruiting patients are expected to be 120 .

\section{Data analysis plan}

A detailed statistical analysis plan will be completed prior to the locking of the study database.

The proportion of ctDNA in circulating free DNA is measured as ctDNA\%. We calculate ctDNA half-life by quantified mean percentage of ctDNA\% in each time point. Assuming an exponential decay model, when the natural logarithm of the plasma ctDNA concentration is plotted against time, a straight line with a slope of $-k$ will be seen. The half-life is determined using the equation of half-life $=0.693 / \mathrm{k} .{ }^{19}$ The slope of the resulting plot of the natural logarithms against time is calculated by linear regression using the SigmaStat 3.0 software (SPSS, Chicago, IL).

\section{Duration of study}

The study is planned to run for 3 years. The expected end date for recruitment is September 2017. Mutation data detected by cSMART will be carrying out as patients recruited at the same time, which is expected to be completed by December 2017. We expect to have completed our primary data analysis with a view to dissemination of results by January 2018, results from the study will be disseminated via presentations at scientific meetings and publications in peer-reviewed journals. After a 3 years' follow-up, we will consider the study complete.

\section{ETHICS AND DISSEMINATION}

Ethical approval has been obtained from the Peking University People's Hospital Medical Ethics Committee (2016PHB156-01). Results will be disseminated through presentations at national and international scientific meetings, publications in peer-reviewed journals and public events involving the local administrations of the cities where the study participants are resident.

\section{Safety of participants}

The only safety concern is that of potential additional venipuncture in patients; the risks of this are of minor harm.

\section{Study management}

The study is managed by the thoracic surgery department in Peking University People's Hospital, Beijing, China.

\section{Sponsorship}

The study is sponsored by Peking university People's Hospital and Berry Genomics Corp.

Acknowledgements The authors wish to acknowledge the contribution of Yun Wang, Yanyan Hou and Dr Kaize Zhong and Dr Qi Huang of Peking University People's Hospital for assistance with sample collection and reservation.

Contributors $\mathrm{KC}$ and $\mathrm{HZ}$ contributed equally to this work as first authors. $\mathrm{KC}$, LTW and JW were involved in study conception. KC, FY and HG were involved in obtaining funding. TW and YS were involved in development of CSMART technique. $\mathrm{KC}, \mathrm{HZ}$ were involved in development of the statistical analysis plan. $\mathrm{KC}, \mathrm{HZ}, \mathrm{FY}, \mathrm{HG}$, TW, LTW and JW were involved in protocol development. KC, HZ, TW were involved in manuscript drafting. All authors have reviewed and approve the final version.

Funding This study is supported by the National Natural Science Foundation of China (No.81602001) and Peking University People's Hospital Research and Development Funds (RDY2016-03).

Competing interests None declared.

Patient consent Obtained.

Ethics approval Ethical approval has been obtained from the Peking University People's Hospital Medical Ethics Committee.

Provenance and peer review Not commissioned; externally peer reviewed.

Data sharing statement Unpublished data will be available from the corresponding author by direct application.

Open Access This is an Open Access article distributed in accordance with the Creative Commons Attribution Non Commercial (CC BY-NC 4.0) license, which permits others to distribute, remix, adapt, build upon this work non-commercially, and license their derivative works on different terms, provided the original work is properly cited and the use is non-commercial. See: http://creativecommons.org/ licenses/by-nc/4.0/ 
(c) Article author(s) (or their employer(s) unless otherwise stated in the text of the article) 2018. All rights reserved. No commercial use is permitted unless otherwise expressly granted.

\section{REFERENCES}

1. Wan JCM, Massie C, Garcia-Corbacho J, et al. Liquid biopsies come of age: towards implementation of circulating tumour DNA. Nat Rev Cancer 2017; 17:223-38.

2. Mok T, Wu YL, Lee JS, et al. Detection and dynamic changes of EGFR mutations from circulating tumor DNA as a predictor of survival outcomes in NSCLC patients treated with firstline intercalated erlotinib and chemotherapy. Clin Cancer Res 2015;21:3196-203.

3. Rosell R, Karachaliou N. Lung cancer: using ctDNA to track EGFR and KRAS mutations in advanced-stage disease. Nat Rev Clin Oncol 2016;13:401-2.

4. Mao X, Zhang Z, Zheng X, et al. Capture-based targeted ultradeep sequencing in paired tissue and plasma samples demonstrates differential subclonal ctDNA-releasing capability in advanced lung cancer. J Thorac Oncol 2017;12:663-72.

5. Newman AM, Bratman SV, To J, et al. An ultrasensitive method for quantitating circulating tumor DNA with broad patient coverage. Nat Med 2014;20:548-54.

6. Garcia-Murillas I, Schiavon G, Weigelt B, et al. Mutation tracking in circulating tumor DNA predicts relapse in early breast cancer. Sci Transl Med 2015;7:302ra133.

7. Lo YM, Zhang J, Leung TN, et al. Rapid clearance of fetal DNA from maternal plasma. Am J Hum Genet 1999;64:218-24.

8. Diehl F, Schmidt K, Choti MA, et al. Circulating mutant DNA to assess tumor dynamics. Nat Med 2008;14:985-90.
9. Heitzer E, Ulz P, Geigl JB. Circulating tumor DNA as a liquid biopsy for cancer. Clin Chem 2015;61:112-23.

10. Vansteenkiste J, Crinò L, Dooms $\mathrm{C}$, et al. 2nd ESMO consensus conference on lung cancer: early-stage non-small-cell lung cancer consensus on diagnosis, treatment and follow-up. Ann Oncol 2014;25:1462-74.

11. Xie Y, Minna JD. A lung cancer molecular prognostic test ready for prime time. Lancet 2012;379:785-7.

12. Tie J, Wang $Y$, Tomasetti $C$, et al. Circulating tumor DNA analysis detects minimal residual disease and predicts recurrence in patients with stage II colon cancer. Sci Trans/ Med 2016;8:346ra92.

13. Chen K, Zhang J, Guan T, et al. Comparison of plasma to tissue DNA mutations in surgical patients with non-small cell lung cancer. J Thorac Cardiovasc Surg 2017;154:1123-31.

14. Wang Z, Cheng G, Han X, et al. Application of single-molecule amplification and resequencing technology for broad surveillance of plasma mutations in patients with advanced lung adenocarcinoma. J Mol Diagn 2017;19:169-81.

15. Lv W, Wei X, Guo R, et al. Noninvasive prenatal testing for Wilson disease by use of circulating single-molecule amplification and resequencing technology (cSMART). Clin Chem 2015;61:172-81.

16. An SJ, Chen ZH, Su J, et al. Identification of enriched driver gene alterations in subgroups of non-small cell lung cancer patients based on histology and smoking status. PLoS One 2012;7:e40109.

17. Shi Y, Au JS, Thongprasert S, et al. A prospective, molecular epidemiology study of EGFR mutations in Asian patients with advanced non-small-cell lung cancer of adenocarcinoma histology (PIONEER). J Thorac Oncol 2014;9:154-62.

18. Chen KZ, Lou F, Yang F, et al. Circulating Tumor DNA detection in early-stage non-small cell lung cancer patients by targeted sequencing. Sci Rep 2016;6:31985.

19. To EW, Chan KC, Leung SF, et al. Rapid clearance of plasma Epstein-Barr virus DNA after surgical treatment of nasopharyngeal carcinoma. Clin Cancer Res 2003;9:3254-9. 